\title{
FORMAS DE POTÁSSIO EM SOLOS DE VÁRZEA E SUA DISPONIBILIDADE PARA O FEIJ OEIRO(1)
}

\author{
M. R. VILLA ${ }^{(2)}$, L. A. FERNANDES ${ }^{(3)} \&$ V. FAQUIN ${ }^{(4)}$
}

\begin{abstract}
RESUMO
0 presente estudo teve como objetivos caracterizar as for mas de $\mathrm{K}$ em solos de várzea e 0 aproveitamento dessas formas pela planta, avaliar a resposta do feijoeiro à aplicação desse nutriente nesses solos e determinar os seus níveis críticos nos solos e nas plantas. 0 delineamento experimental utilizado foi o inteiramente casualizado, em esquema fatorial 4 x 5, com quatro repetições, sendo quatro solos de várzea [Gleissolo Melânico (GM), Gleissolo Háplico (GX), Neossolo Flúvico (RU) e Organossolo Méssico (OY)] e cinco níveis de saturação por $\mathrm{K}$ da CTC potencial (natural, 4, 6, 8 e 10\%). Foram cultivadas quatro plantas de feijoei ro (Phaseol us vulgaris L.) por vaso até a produção de grãos. Amostras dos solos foram coletadas para a determinação do K total, não-trocável, trocável, em solução, disponível pelos extratores Mehlich-1 e Resina e medidas da relação Quantidade/l ntensidade de K. Os teores de K total eK não-trocável foram maiores nos solos OY e RU, enquanto os teores de $K$ trocável e $K$ em solução foram maiores nos solos OY e GM. As saturações críticas por $K$ da CTC potencial foram de 5,0, 4,1, 8,7 e 5,3\%, enquanto os níveis críticos de $K$ foliar foram de 17,9, 30,5, 16,8 e 14,6 $\mathrm{g} \mathrm{kg}^{-1}$, para os solos OY, GM, GX e RU, respectivamente.
\end{abstract}

Termos para indexação: extratores de potássio, relação solo-planta, química do solo.

\footnotetext{
(1) Parte da Tese de Mestrado do primeiro autor. Recebido para publicação em dezembro de 2001 e aprovado em julho de 2004.

(2) Pós-Graduando do Departamento de Ciência do Solo, Universidade Federal de Lavras - UFLA. Caixa Postal 37, CEP 37200-000 Lavras (MG).E-mail: mvila@ufla.br

(3) Professor do Instituto de Ciências Agrárias, Universidade Federal de Minas Gerais - UFMG. Caixa Postal 135, CEP 39404-006 Montes Claros (MG). E-mail: larnaldo@ufmg.br

(4) Professor do Departamento de Ciência do Solo, UFLA. Bolsista do CNPq. E-mail: vfaquin@ufla.br
} 


\title{
SUMMARY: POTASSIUM FORMS IN LOWLAND SOILS AND AVAILABILITY TO COMMON BEAN
}

\begin{abstract}
The present study had as objectives to characterize K forms, plant use of these forms and to eval uatethe common bean (Phaseol us vulgaris L.) response to $K$ application in four lowland soils. It was al so sought to determine the critical levels for $\mathrm{K}$ in soils and plant. The experimental design was completely randomized in a $4 \times 5$ factorial scheme with four replications. The treatments were a combination of four lowl and soils [Mesic Organosoil (OY), Melanic Gleysoil (GM), Haplic Gleysoil (GX), and Fluvic Neosoil (RU)] and five K CEC saturation levels (native, 4, 6, 8, and $10 \%$ ). Four bean plants per pot werecultivated until grain production. Soil samples were analyzed for total K, non-exchangeable K, exchangeable K, soil solution K, available K by Mehlich- 1 and K extractable by a cation exchange resin. TheK quantity/ intensity ratio was also determined. The concentration of total and non-exchangeableK werehigher in OY and RU soils, while exchangeableK and soil solution $\mathrm{K}$ were higher in $\mathrm{O}$ and $\mathrm{GH}$ soils. The critical $\mathrm{K}$ saturation of $\mathrm{CEC}$ were 5.0 , 4.1, 8.7, and $5.3 \%$ and thecritical foliar K levels were $17.9,30.5,16.8$, and $14.6 \mathrm{mg} \mathrm{kg}^{-1}$ for OY, GM, GX, and RU soils, respectively.
\end{abstract}

Index terms: potassium extractors, soil - plant relationship, soil chemistry.

\section{NTRODUÇÃO}

O K no solo apresenta-se em diversas formas, muitas das quais não-disponíveis às plantas. Considerando sua disponibilidade para as plantas, o $\mathrm{K}$ do solo pode ser classificado em quatro categorias, que seguem uma ordem crescente de disponibilidade: estrutural (mineral), não-trocável ou dificilmente disponível, trocável e em solução, que, somadas, fornecem o K total (Tisdale \& N elson, 1993). Como essas frações encontram-se em equilíbrio dinâmico (Sparks, 1980), extratores químicos específicos têm sido utilizados nas suas quantificações, tendo como vantagem permitir a comparação das formas de $\mathrm{K}$ sob diferentes condições.

O K não-trocável compreende o K adsorvido nas entrecamadas de minerais de argila 2:1 e uma parte do $\mathrm{K}$ contido em minerais primários de mais fácil intemperização (Mielniczuk \& Selbach, 1978), comumente extraído com $\mathrm{HNO}_{3}$ fervente (K nudsen et al., 1982). A diferença entre a fração assim extraída e a fração trocável constitui a fração de K não-trocável do solo, a qual representa a porção do $\mathrm{K}$ do sol o que será li berada a médio prazo.

$A$ fração de $K$ solúvel é constituída pelo $K$ que se encontra na solução do solo, em dado momento, sob condições normais de umidade e livre de forças de adsorção (Reitemeier, 1951). Essa fração apresenta uma pequena participação em relação ao K total do solo, e a sua avaliação apenas demonstra a disponibilidade momentânea de $\mathrm{K}$ para as plantas (Tisdale \& Nelson, 1993). Contudo, sua importância pode, às vezes, ser significativa, visto que, em determinadas situações, o K da solução éo principal fator a influenciar a absorção deste nutriente pelas plantas (Gianelo \& Mielniczuk, 1981).
Para mel horar a avaliação da disponibilidade de $K$ para as plantas, é importante que se tenha conhecimento não só dos fatores quantidade (Ktrocável) e intensidade (K-solução), mas também da rel ação entre esses dois fatores que constitui o Poder Tampão de Potássio (PTK) do solo, ou seja, a capacidade que o solo tem em manter certa concentração de K na solução, quando da absorção pelas plantas ou de perdas ocorridas no sol o (Goedert et al., 1975; Mielniczuk, 1982).

A relação quantidade/intensidade(Q/I) foi desenvolvida por Beckett (1964), após confirmação da Lei da Relação estabelecida por Schoefield (1947), citado por Goedert et al. (1975), que introduziu o conceito de potencial químico como propriedade relacionada com a disponibilidade de nutrientes às plantas. O conceito de quociente de atividade de $\mathrm{K}$ (QAK), dado pela relação $a K /(\mathrm{aCa})^{0,5}$, é uma medida do potencial químico do $\mathrm{K}$ em relação ao do cálcio presentena solução do sol o (Beckett, 1964). Segundo esse autor, a disponibilidade de $\mathrm{K}$ no solo ou sua troca pelo $\mathrm{Ca}$ depende do potencial de $\mathrm{K}$ e do potencial de $\mathrm{Ca}$. Como o $\mathrm{Ca}$ e o Mg apresentam propriedades de troca muito semelhantes, eles podem ser considerados como sendo uma única espécie e, dessa maneira, o QAK pode ser calculado como aK/(aCa +aMg) ${ }^{0,5}$ (Beckett, 1964).

Segundo Beckett \& Nafady (1967), quandose está estudando o termo quantidade em relação ao QAK, este deve ser medido em termos de $\Delta \mathrm{K}$. Este termo representa a quantidade de $K$ adsorvido $(+\Delta K)$ ou dessorvido $(-\Delta K)$ pel o sol o quando em equilíbrio com soluções de diferentes QAK. Estudando solos de Oxford, Beckett (1964) verificou quea tendência dos gráficos da relação Q/I para os solos estudados era a mesma, uma parte superior linear e uma inferior na forma de curva assintótica em relação ao eixo $\Delta K$. 
Como a inclinação da parte reta $\Delta \mathrm{K} / \Delta \mathrm{QAK}$ representa o poder tampão de $K$ do solo (PTK), em concentrações baixas deK trocável, maiores val ores serão obtidos mediante o aumento do coeficiente de seletividade, ou seja, maior retenção deK em relação ao Ca mais Mg (Mielniczuk, 1978). A parte inferior do gráfico representa a quantidade total de K trocável no solo, sendo oK preferencial (posições "e"e "i") dado pela diferença entre os valores de $\Delta K$ correspondente à extremidade da região curva e à extrapolação da região reta do gráfico. O K planar, destemodo, éa diferença entre o K trocável total eo K preferencial (Mielniczuk, 1978; Beckett \& Nafady, 1967).

Tais medidas obtidas por meio da curva Q/I são muito variáveis de acordo com as características físico-químicas e mineralógicas dos solos. A composição do complexo sortivo ou tipo de argila predominante é que vai determinar se o $\mathrm{K}$ vai ser mais ou menos facilmente liberado para a solução.

O presenteestudo teve como objetivos caracterizar as formas deK nosol o, avaliar a resposta do feijoeiro à aplicação desse nutriente em solos de várzea e determinar os níveis críticos de K nesses sol os e nas plantas.

\section{MATERIAL E MÉTODOS}

O experimento foi realizado em condições de casa de vegetação do Departamento de Ciência do Solo da Universidade Federal de Lavras - UFLA, Lavras (MG). Utilizaram-se amostras da camada de 0,00,2 m de quatro sol os de várzea: Gleissolo Melânico (GM), Gleissolo Háplico (GX), N eossolo Flúvico (RU) e Organossolo Méssico (OY), coletados na camada superficial $(0-20 \mathrm{~cm})$, em uma única várzea não sistematizada no muniápio de Lavras (MG). A coleta das amostras e o experimento foram realizados no período de 1996 a 1998.

Inicialmente, amostras de $4 \mathrm{~kg}$ dos solos foram incubadas por 30 dias, mantendo-se a umidade próxima à capacidade campo, $\mathrm{com} \mathrm{CaCO}_{3} \mathrm{e} \mathrm{MgCO}_{3}$ p.a, na relação equivalente de $\mathrm{Ca:Mg}$ de 4:1, para elevar a saturação por bases a $50 \%$. Após esse período, os solos foram secos ao ar e analisados quanto aos seus atributos químicos, físicos e mineralógicos (Quadro 1).

O delineamento experimental utilizado foi o intei ramente casualizado, em esquema fatorial $4 \times 5$, sendo quatro solos de várzea (GM, GX, RU e OY) e cinco níveis de saturação por $\mathrm{K}$ da CTC potencial (natural, 4, 6, 8 e 10\%), com quatro repetições. As doses de $\mathrm{K}$ necessárias para atingir os níveis de saturação da CTC potencial foram de: 28,1, 112,3, 196,6 e $280,8 \mathrm{mg} \mathrm{dm}^{-3}$ de K, respectivamente, para o solo OY; 78,0, 187,2, 296,4 e 405,6 $\mathrm{mg} \mathrm{dm}^{-3}$ de K, para o solo GM; 46,0, 92,4 ,138,8 e 185,3 $\mathrm{mg} \mathrm{dm}^{-3}$ de K, para o solo GX, e 59,9, 126,4, 195,9 e 265,2 $\mathrm{mg} \mathrm{dm}^{-3}$ de K, para o solo RU. No tratamento saturação por $\mathrm{K}$ natural, esta correspondeu a 3,3, 2,6, 1,9 e 2,4 \%, para os solos OY, GM, GX e RU, respectivamente, val ores cal culados com base nos dados apresentados no quadro 1.

J untamente com os níveis de $\mathrm{K}$ referentes aos tratamentos, foi aplicada uma adubação básica de semeadura com $80 \mathrm{mg}$ de $\mathrm{N}, 300 \mathrm{mg}$ de $\mathrm{P}, 40 \mathrm{mg}$ de $\mathrm{S}, 0,5 \mathrm{mg}$ de $\mathrm{B}, 1,5 \mathrm{mg}$ de Cu e $5 \mathrm{mg} \mathrm{dm}^{-3}$ de $\mathrm{Zn}$ de solo, na forma de reagentes p.a.: $\mathrm{KNO}_{3}, \mathrm{KH}_{2} \mathrm{PO}_{4}$, $\mathrm{K}_{2} \mathrm{SO}_{4}, \mathrm{H}_{3} \mathrm{PO}_{4}, \mathrm{NH}_{4} \mathrm{NO}_{3}, \mathrm{NH}_{4} \mathrm{H}_{2} \mathrm{PO}_{4}, \mathrm{Ca}\left(\mathrm{H}_{2} \mathrm{PO}_{4}\right)_{2}$, $\mathrm{H}_{3} \mathrm{BO}_{3}, \mathrm{CuSO}_{4}, \mathrm{ZnSO}_{4} \mathrm{e}\left(\mathrm{NH}_{4}\right)_{2} \mathrm{SO}_{4}$. Essas amostras permaneceram em incubação por 30 dias.

No final desse período, subamostras de $1 \mathrm{~kg}$ de cada vaso foram retiradas para análises químicas e os $3 \mathrm{~kg}$ restantes foram colocados em vasos com capacidade de $4 \mathrm{dm}^{3}$ para o cultivo de feijoeiro (Phaseol us vulgaris L. cv. Carioca - MG). Duranteo período experimental, foram cultivadas quatro plantas por vaso, as quais receberam adubações de coberturas com $\mathrm{N}$ na forma de $\mathrm{NH}_{4} \mathrm{NO}_{3}$, na dose de $175 \mathrm{mg} \mathrm{dm}^{-3} \mathrm{de} \mathrm{N}$, para a dose zero de $\mathrm{K}$, e $200 \mathrm{mg} \mathrm{dm}^{-3} \mathrm{de} \mathrm{N}$, para os demais tratamentos, parcelados em três vezes, até à época do préflorescimento.

Foram col etadas amostras de sol o imediatamente após a aplicação dos tratamentos de saturação por $K$ e após o cultivo do feijoeiro, para determinar as formas de K, ou seja, $K$ total (K tot), K não-trocável (Knt), K trocável (Kt) e K na solução (Ksol).

Os teores totais no sol o foram determinados após digestão lenta em banho de areia com $\mathrm{HF}, \mathrm{HClO}_{4} \mathrm{e}$ $\mathrm{HNO}_{3}$ concentrados (Knudsen et al., 1982). O K trocável foi determinado pelo $\mathrm{NH}_{4} \mathrm{OAc} 1 \mathrm{~mol} \mathrm{~L}-1$ pH 7,0 (Mielniczuk, 1978), e o K disponível pelo Mehlich-1 (Embrapa, 1997) e pela resina de troca iônica (Raij et al., 1987). A fração de K não-trocável foi obtida pela diferença entre a fração extraída com $\mathrm{HNO}_{3} 1 \mathrm{~mol} \mathrm{~L}^{-1}$ por 10 minutos de fervura e aquela obti da com $\mathrm{NH}_{4} \mathrm{OAc} 1 \mathrm{~mol} \mathrm{~L}^{-1} \mathrm{pH} 7,0$. O K da solução foi avaliado conforme Miel niczuck (1978).

As quantidades de $K$, consideradas liberadas de formas não prontamente disponíveis (Knt) e de formas prontamente disponíveis $(\mathrm{K} t)$ durante $\mathrm{o}$ cultivo, foram calculadas por diferença entre as concentrações inicial mente presentes no sol o e após o cultivo, bem como pel ototal absorvido pel o feijoei ro (Quadro 2).

As medidas da relação quantidade/intensi dade (Q/ I) foram determinadas nas subamostras após a incubação com calcário, conforme método descrito por Mielniczuk (1978). A adsorção ( $\Delta \mathrm{K}$ positivo) ou dessorção ( $\Delta K$ negativo) de $K$ nos solos foram determinadas pela diferença entre a concentração do el emento nas soluções iniciais e de equilíbrio. $O$ quociente de atividade de K (QAK) foi determinado pela relação de atividade de acordo com a equação: $\mathrm{QAK}=\mathrm{aK}^{+} /\left(\mathrm{aCa}^{2+}+\mathrm{aMg}^{2+}\right)^{0,5}$. 
Quadro 1. Atri butos químicos, físicos e mi neralógicos dos solos: Gleissolo Háplico (GX), Neossolo Flúvico (RU), Gleissolo Melânico (GM) e Organossolo Mésico (OY) ${ }^{(1)}$

\begin{tabular}{|c|c|c|c|c|c|c|c|c|c|c|c|c|c|}
\hline Solo & $\mathbf{p H}$ & $\mathbf{P}$ & $\mathbf{K}$ & $\mathrm{Ca}^{2+}$ & $\mathbf{M g}^{2+}$ & $\mathrm{Al}^{3+}$ & $\mathbf{H}+\mathbf{A l}$ & $\mathbf{t}$ & $\mathbf{T}$ & $\mathbf{m}$ & $\mathbf{V}$ & CMAP & PR \\
\hline & & \multicolumn{2}{|c|}{$-\mathrm{mg} \mathrm{dm}^{-3}$} & \multicolumn{6}{|c|}{$\mathrm{cmol}_{\mathrm{c}} \mathrm{dm}^{-3}$} & \multicolumn{2}{|c|}{$\%$} & $\mathrm{mg} \mathrm{kg}^{-1}$ & $m g L^{-1}$ \\
\hline $\mathrm{GX}$ & 4,5 & 3 & 36 & 0,6 & 0,2 & 1,1 & 6,3 & 2,0 & 7,2 & 55 & 12 & 648 & 28 \\
\hline $\mathrm{RU}$ & 4,7 & 3 & 103 & 2,7 & 1,1 & 0,2 & 4,5 & 4,3 & 8,6 & 5 & 47 & 689 & 27 \\
\hline $\mathrm{GM}$ & 4,7 & 8 & 51 & 0,7 & 0,2 & 1,6 & 13,7 & 2,6 & 14,7 & 61 & 7 & 2.404 & 2 \\
\hline OY & 4,6 & 6 & 76 & 1,6 & 1,2 & 0,9 & 9,8 & 3,9 & 12,8 & 23 & 23 & 1.051 & 16 \\
\hline
\end{tabular}

\begin{tabular}{|c|c|c|c|c|c|c|c|c|}
\hline & $\begin{array}{l}\text { Areia } \\
\text { grossa }\end{array}$ & $\begin{array}{c}\text { Areia } \\
\text { fina }\end{array}$ & Silte & Argila & $\begin{array}{l}\text { Matéria } \\
\text { orgânica }\end{array}$ & $\begin{array}{c}\text { Densidade do } \\
\text { solo }\end{array}$ & $\begin{array}{l}\text { Densidade de } \\
\text { partícula }\end{array}$ & $\begin{array}{l}\text { Superfíci } \\
\text { específic }\end{array}$ \\
\hline GX & 30 & 611 & 179 & 180 & 31 & 1,0 & 2,7 & 121 \\
\hline $\mathrm{RU}$ & 0 & 179 & 471 & 350 & 31 & 0,8 & 2,8 & 137 \\
\hline GM & 10 & 230 & 390 & 370 & 230 & 0,4 & 1,8 & 335 \\
\hline OY & 0 & 120 & 570 & 310 & 44 & 0,6 & 2,1 & 181 \\
\hline
\end{tabular}

\begin{tabular}{|c|c|c|c|c|c|c|c|c|c|c|}
\hline & $\mathrm{SiO}_{2}$ & $\mathbf{A l}_{2} \mathbf{O}_{3}$ & $\mathrm{Fe}_{2} \mathrm{O}_{3}$ & $\mathrm{TiO}_{2}$ & $\mathrm{P}_{2} \mathrm{O}_{5}$ & $F e_{d}$ & $F e_{o}$ & $F e_{o} / F e_{d}$ & G b & Ct \\
\hline $\mathrm{GX}$ & 84,2 & 112,2 & 13,0 & 6,2 & 0,1 & 1,8 & 0,6 & 0,33 & 19 & 65 \\
\hline $\mathrm{RU}$ & 209,3 & 221,1 & 74,5 & 9,3 & 0,5 & 12,3 & 0,5 & 0,04 & 49 & 143 \\
\hline GM & 126,1 & 175,5 & 13,3 & 5,5 & 1,5 & 2,3 & 0,6 & 0,26 & 53 & 48 \\
\hline OY & 243,4 & 247,1 & 42,8 & 7,3 & 0,5 & 5,3 & 0,5 & 0,09 & 57 & 121 \\
\hline
\end{tabular}

(1) $\mathrm{pH}=\mathrm{pH}$ em água; $\mathrm{P}=$ fósforo pelo $\mathrm{Mehlich}-1 ; \mathrm{PR}=\mathrm{P}$ remanescente; $\mathrm{CMAP}=$ capacidade máxima de adsorção de $\mathrm{P} ; \mathrm{SiO}_{2}, \mathrm{Al}_{2} \mathrm{O}_{3}$, $\mathrm{Fe}_{2} \mathrm{O}_{3}, \mathrm{TiO}_{2}$ e $_{2} \mathrm{O}_{5}$ (E mbrapa, 1997); $\mathrm{Fe}_{\mathrm{d}}=$ ferro ditionito, $\mathrm{Fe}_{\mathrm{o}}=$ ferro oxalato, $\mathrm{Fe}_{\mathrm{o}} / \mathrm{Fe}_{\mathrm{d}}=$ atividade dos óxidos de ferro, $\mathrm{Ct}=$ caulinita e $\mathrm{Gb}=$ gibsita.

Quadro 2. Teores médios de Ktotal (Ktot), K não-trocável (Knt), K trocável (Kt), K solução (Ksol) e K disponível pelos extratores Mehlich-1 (K-M1) e Resina (K-Res) para quatro solos de várzea após a incubação com calcário, sem aplicação de $K$

\begin{tabular}{|c|c|c|c|c|c|c|}
\hline Solo & Ktot & Knt & $\mathbf{K t}$ & Ksol & K-M 1 & K-Res \\
\hline OY & $9.369,8$ & $95,9(1,0)^{(1)}$ & $108,3(1,2)$ & $104,1(1,1)$ & $136,2(1,5)$ & $118,5(1,3)$ \\
\hline GM & $1.039,8$ & $65,3(6,3)$ & $115,8(11,1)$ & $102,6(9,9)$ & $111,2(10,7)$ & $105,5(10,2)$ \\
\hline $\mathrm{GX}$ & $1.123,1$ & $23,6(2,1)$ & $24,1(2,2)$ & $36,9(3,3)$ & $44,5(4,0)$ & $33,7(3,0)$ \\
\hline RU & $8.481,3$ & $237,6(2,8)$ & $64,7(0,8)$ & $69,0(0,8)$ & $90,0(1,1)$ & $73,0(0,9)$ \\
\hline
\end{tabular}

(1) Número entre parênteses representa a contribuição percentual de cada fração de K para o Ktot.

O coeficiente de atividade (f), para calcular a atividade do $\mathrm{K}\left(\mathrm{a}=\mathrm{f} \times \mathrm{c}_{\mathrm{i}}\right)$, foi determinado pela fórmula de Debye-Huckel, sendo considerados os íons em suas formas livres.

As medidas da relação Q/I de K decada solo foram determinadas, a partir dos dados das concentrações de $\mathrm{K}^{+}, \mathrm{Ca}^{2+}$ e $\mathrm{Mg}^{2+}$ em cada nível de $\mathrm{K}$ usado nas diferentes soluçôes de equilíbrio com os solos. Por meio de equações ajustadas para cada solo, foi construída "a curva" que relaciona o $\Delta \mathrm{K}$ e o QAK e estimaram-se os valores de $\mathrm{K}$ em equilíbrio com a solução, K planar, preferencial, poder tampão de K (PTK), quociente de atividade de equilíbrio (QAKe) e concentração de $K$ na solução do solo $(\mathrm{Ke})$. Os valores deK planar, QAKeePTK foram obtidos por meio do ajuste de uma equação de regressão do tipo linear para os pontos obtidos a partir de concentrações crescentes de K na solução inicial e 
$3 \mathrm{~g}$ de solo. O QAKe foi considerado o ponto onde o $\Delta \mathrm{K}$ foi igual a zero, o $\mathrm{K}$ planar como sendo o intercepto da curva ao eixo $\Delta \mathrm{K}$, ou seja, quando QAK $=0$, e oPTK, pela declividade da reta.

Para obter o K em equilíbrio com a solução, ajustou-se equação de regressão do tipo quadrática aos pontos obtidos sem adição de K na solução inicial e com redução na quantidade de solo $(1,5 ; 0,8 ; 0,4$ e $0,2 \mathrm{~g}$ de solo), sendo o val or do $\mathrm{K}$ em equilíbrioaquele quando $\mathrm{QAK}=0$, ou seja, o intercepto da região curva da figura ao eixo $\Delta K$. Pela diferença entreo $K$ planar e o K em equilíbrio com a solução, obteve-se oK preferencial. A concentração de equilíbrio de K na solução do sol o foi obtida multiplicando-se o valor de QAKe pela atividade de $\left(\mathrm{Ca}^{2+}+\mathrm{Mg}^{2+}\right)^{0,5}$.

$\mathrm{Na}$ época do pleno florescimento, amostrou-se uma folha recém-madura de cada planta ( 4 folhas por vaso), para a determinação dos teores de K no tecido foliar, conforme Malavolta et al. (1997). No final do ciclo, todas as plantas foram col hidas, e os grãos foram secos em estufa a $65-70 \stackrel{\circ}{\circ}$, para obtenção da matéria seca.

As variáveis estudadas foram submetidas à analise de variância e estudos de regressão. Pelo ajuste de equações de regressão entre a produção de grãos e as saturações por K da CTC potencial estabel ecidas nos tratamentos, foram estimadas as saturações por K necessárias para atingir a produção máxima e para atingir 90 \% da produção máxima em cada solo. Substituindo esses valores nas equações que relacionam o $\mathrm{K}$ disponível nos solos pelos extratores Mehlich-1 e resina, conforme as saturações por $\mathrm{K}$ estabelecidas nos tratamentos, estimaram-se os níveis críticos inferiores de $\mathrm{K}$ disponível para $90 \%$ da produção máxima para os diferentes solos. Da mesma maneira, estimaramse os níveis críticos inferiores de $\mathrm{K}$ nos tecidos das folhas do feijoeiro, coletadas na época do florescimento, pela substituição das saturações por K para $90 \%$ da produção máxima de grãos, nas equações de regressão que relacionaram as saturações por $K$ com os teores de $K$ nas fol has do feijoeiro na época do florescimento.

\section{RESULTADOS E DISCUSSÃO}

Com relação ao $\mathrm{K}$ total (K tot), observa-se que os teores diferiram entre os solos estudados (Quadro 2), variando de 1.039,8 $\mathrm{mg} \mathrm{dm}^{-3}$, no Gleissolo Melânico (GM ), a 9.369,8 $\mathrm{mg} \mathrm{dm}^{-3}$, no Organossolo Mésico (OY). Prezotti \& Defelipo (1987), trabal hando com várias classes de solos do estado de Minas Gerais, também encontraram uma ampla variação para os teores de $\mathrm{K}$ total.

Exceto para o Neossol o F lúvico (RU), em que o K não-trocável (Knt) foi a fração que mais contribuiu para oK tot, os teores mais el evados de K tot parecem estar ligados aos teores mais el evados da fração trocável e disponível. N o entanto, para o solo GM e GX, a participação elevada das formas de $K$ mais prontamente disponíveis (K trocável e disponível) para o Ktot não indica el evados teores do nutriente no solo, pois, na verdade, esta contribuição está relacionada com os teores relativamente baixos obtidos para oK tot desses sol os (Quadro 2).

Para o Knt, observaram-se grandes diferenças entre os solos, sendo os mai ores val ores observados para os solos OY e RU, mostrando, neste caso, que esta forma de reserva de K está relacionada com os teores mais el evados de silte (Quadro 1) e com oK tot (Quadro 2) desses solos. Deve-se ressaltar que, nos solos OY, GM e GX, a fração não-trocável de K é até mesmo inferior àquela trocável, indicando que a extração com $\mathrm{HNO}_{3} 1 \mathrm{~mol} \mathrm{~L}^{-1}$ a quente pode não ser o melhor método para avaliar a reserva de $\mathrm{K}$ em certos solos. Esse comportamento provavel mente é um dos motivos pelo qual a classificação do K do solo em trocável e não-trocável, em determinadas situações, não se tem mostrado adequada (Silva et al., 2000).

A menor capacidade de extração de $\mathrm{K}$ pelo $\mathrm{HNO}_{3}$ $1 \mathrm{~mol} \mathrm{~L}^{-1}$ a quente em comparação ao acetato de amônio pode estar relacionada com a presença de micas resistentes ao intemperismo na fração argila de alguns solos. Conforme Rich (1968), estas micas podem no processo de intemperização conter ilhas de hidróxi-Al entrecamadas, que, sob condições de pH mais el evado, como no caso do acetato deamônio, são precipitadas, ocorrendo assim a extração do K presente nas entrecamadas.

Os quatro solos apresentaram teores de $\mathrm{K}$ na solução (K sol) acima de $11,7 \mathrm{mg} \mathrm{dm}^{-3}$ (Quadro 2), teores estes considerados como suficiente para o desenvol vimento vegetal (Raij, 1981). Por outrolado, com exceção do GM, a baixa CTC efetiva dos solos (Quadro 1) favorece a manutenção de teores de K na solução do solo bastante superiores àquele considerado ideal, como pode ser observado pela significativa participação dessa fração para o Ktot, principalmente para os sol os GM eGX. No entanto, graças a baixa CTC do solo GX (Quadro 1), esse apresenta maior susceptibilidadeàs perdas deK por lixiviação em relação aos demais.

Os valores de $\mathrm{K}$ disponível pelos extratores Mehlich-1 e Resina foram muito próximos (Quadro 2), apresentando um coeficiente de correlação $(r)$ de $0,99(P<0,01)$ para os solos em estudo. Esses resultados mostram que o K ocupa, no complexo, posição de troca acessível aos dois extratores testados. Seoteor deK disponível acima de $70 \mathrm{mg} \mathrm{dm}^{-3}$ pel o M ehlich-1 for considerado como bom para a maioria das culturas (CFSEMG, 1999), apenas o GX apresentaria teores deK em teor abaixo do adequado (Quadro 2). I sto porque esse solo apresenta baixa CTC efetiva e baixos teores de silte 
e argila (Quadro 1), os quais influenciam os teores disponíveis e trocáveis desse nutriente.

Para as medidas da relação Q/I (Quadro 3), verifica-se que o solo $\mathrm{GX}$ apresentou praticamente todo o seu $K$ sujeito a entrar em equilíbrio com a solução (K planar + K preferencial), adsorvido na posição preferencial de adsorção (K preferencial), o que explica seu menor teor de $\mathrm{K}$ em solução (Quadro 2), uma vez que o K adsorvido na posição planar ( $K$ planar) é responsável imediato pela manutenção do K em solução. J á os solos OY e GM apresentaram teores praticamentesemel hantes de K sujeito a entrar em equilíbrio com a solução, no entanto, o solo OY apresentou mai or teor deK planar. Dessa forma, embora os teores de $K$ na solução do sol o sejam praticamentesemel hantes (Quadro 2), com o esgotamento dessa forma de $\mathrm{K}$ pelo cultivo ou por lixiviação, o solo OY manterá maior teor de K em solução do solo em relação ao GM, corroborando os val ores de Ke para essa afirmativa.

O conhecimento do teor deK adsorvido na posição planar ou na posição preferencial é de grande importância, quando são feitas comparações dos sol os em relação à disponibilidade de K para as culturas. Tomando por base apenas os teores de $K$ trocável determinados pelos métodos tradicionais, quanto maior o teor de $\mathrm{K}$ trocável no solo, mais $\mathrm{K}$ será mantido na solução do solo. No entanto, tal afirmativa nem sempre será verdadeira, considerando a posição em que se encontra o $\mathrm{K}$ sujeito a equilíbrio (Chaves \& Kinjo 1987).

Os menores valores de atividade de equilíbrio (QAKe) e de atividade de $K$ na solução do solo (Ke) foram observados para o solo GX (Quadro 3). Além disso, pode-se constatar que esse sol otem um menor poder tampão de K (PTK) em relação aos demais solos, o queé conferido pel os seus teores mais baixos de K trocável e em solução (Quadro 2) que se encontram em equilíbrio dinâmico. Portanto, as adubações potássicas nesse solo deverão ser feitas em doses menores e mais freqüentes de forma a minimizar as perdas de $K$ por lixiviação e, até mesmo, o consumo de luxo pelas plantas. Por outro lado, para o solo GM, que apresenta o maior PTK, as adubações poderão ser feitas em doses maiores e mais espaçadas, visto que possui maior capacidade de manter a atividade de K em solução.

O solo OY, embora apresente maior teor de $\mathrm{K}$ planar (Quadro 3) e um teor de K em solução praticamente semel hante à do GM (Quadro 2), apresenta menor poder tampão, indicando que, se o $\mathrm{K}$ for absorvido da solução do solo pelas plantas a médi os elongos prazos, a capacidade de repô-lo será reduzida, de modo que, se nenhuma aplicação for feita, os cultivos sucessivos poderão sofrer deficiência deste nutriente.

Portanto, os solos estudados diferem quanto à capacidade de fornecer $\mathrm{K}$ às plantas e requerem técnicas distintas com relação ao manejo da adubação com esse nutriente. Fica evidente, deste modo, a importância da adoção de práticas que visem aumentar a capaci dade de retenção deK nesses solos.

Com o aumento das doses deK estabelecidas nos tratamentos de saturação, de maneira geral, aumentaram também os teores de $K$ de todas as formas avaliadas (Quadro 4). Com relação ao K trocável, como já era esperado, os maiores teores foram encontrados para os solos OY e GX que mostram CTC potencial mais el evada (Quadro 1), pois estes necessitam demaior quantidade deK para atingir a mesma saturação por este nutriente. Os teores deK na solução (K sol) dos sol os apresentaram a mesma tendência observada para K trocável.

Observou-se que, em todos os sol os estudados, a quantidade de $\mathrm{K}$ liberada durante o cultivo do feijoeiro para alguns níveis de saturação não foi suficiente para atender à demanda por $\mathrm{K}$ da planta (Quadro 5), ocasionando, deste modo, maior quantidade absorvida do que liberada, provavelmente, graças à participação de formas de $K$ não extraídas pelo $\mathrm{HNO}_{3}$ (Nachtigall \& Vahl, 1989; Silva et al., 2000).

Pela correlação positiva obtida entre oK trocável inicial e o K trocável liberado (Quadro 6), verificouseque, quanto maior oteor inicial deK trocável, maior foi sua disponi bilidade para as plantas. Considerações semel hantes foram feitas aoK não-trocável inicial que

Quadro 3. Potássio sujeito ao equilíbrio (Kse), planar (K planar) e preferencial (K pref), quociente de atividade de equilíbrio (QAKe), concentração de equilíbrio de $K$ na solução do solo (Ke) e poder tampão de K (PTK) de quatro solos de várzea

\begin{tabular}{ccccccc}
\hline Solo & Kse & K planar & K pref & QAKe & Ke & PTK \\
\hline & & mmol kg-1 & & $($ mmol L-1) 0,5 & mmol L-1 & $\left(\mathrm{mmol} \mathrm{kg}^{-1}\right) /\left(\mathrm{mmol} \mathrm{L}^{-1}\right)^{0,5}$ \\
OY & 7,3 & 4,1 & 3,2 & 0,65 & 0,77 & 6,3 \\
GM & 7,7 & 3,8 & 3,9 & 0,35 & 0,43 & 11,0 \\
GX & 2,5 & 0,6 & 1,9 & 0,13 & 0,17 & 4,2 \\
RU & 6,0 & 2,1 & 3,9 & 0,21 & 0,31 & 9,9 \\
\hline
\end{tabular}


Quadro 4. Teores médios de Ktotal (Ktot), K não-trocável (Knt), K trocável (Kt), K solução (Ksol), $K$ Mehlich-1 (K-M1) e K Resina (K-Res) antes do cultivo do feijoeiro em cinco níveis de saturação por K da CTC potencial de quatro solos de várzea

\begin{tabular}{|c|c|c|c|c|c|c|c|}
\hline Solo & Tratamento & Ktot & Knt & Kt & Ksol & K-M1 & K-Res \\
\hline & $\%$ & 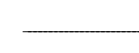 & 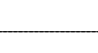 & $r$ & 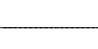 & 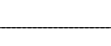 & - \\
\hline \multirow[t]{5}{*}{ OY } & 0 & $9.369,8$ & 95,9 & 108,3 & 104,1 & 136,2 & 118,5 \\
\hline & 4 & $9.203,2$ & 98,6 & 138,8 & 119,1 & 158,0 & 147,7 \\
\hline & 6 & $9.578,1$ & 131,3 & 198,4 & 177,3 & 218,7 & 209,2 \\
\hline & 8 & $9.911,3$ & 163,5 & 251,0 & 230,4 & 278,2 & 292,0 \\
\hline & 10 & $10.119,5$ & 215,2 & 305,1 & 275,2 & 338,0 & 372,5 \\
\hline \multirow[t]{5}{*}{ GM } & 0 & $1.039,8$ & 65,3 & 115,8 & 102,6 & 111,2 & 105,5 \\
\hline & 4 & $1.289,7$ & 94,9 & 180,4 & 170,6 & 166,5 & 169,7 \\
\hline & 6 & $1.373,0$ & 164,7 & 270,5 & 212,4 & 244,7 & 259,0 \\
\hline & 8 & $1.456,3$ & 221,1 & 369,7 & 265,5 & 331,7 & 350,2 \\
\hline & 10 & $1.581,3$ & 246,0 & 473,3 & 408,8 & 399,2 & 434,5 \\
\hline \multirow[t]{5}{*}{$\mathrm{GX}$} & 0 & $1.123,1$ & 23,6 & 24,1 & 36,9 & 44,5 & 33,7 \\
\hline & 4 & $1.317,5$ & 39,6 & 48,2 & 51,8 & 87,7 & 76,5 \\
\hline & 6 & $1.484,1$ & 51,4 & 70,7 & 76,5 & 132,5 & 115,0 \\
\hline & 8 & $1.456,3$ & 57,8 & 88,7 & 106,4 & 167,2 & 149,5 \\
\hline & 10 & $1.456,3$ & 79,3 & 124,8 & 139,2 & 223,7 & 268,2 \\
\hline \multirow[t]{5}{*}{$\mathrm{RU}$} & 0 & $8.481,3$ & 237,6 & 64,7 & 69,0 & 90,0 & 73,0 \\
\hline & 4 & $8.203,6$ & 281,4 & 96,3 & 98,1 & 137,2 & 113,5 \\
\hline & 6 & $8.037,0$ & 274,4 & 144,3 & 140,7 & 193,7 & 178,7 \\
\hline & 8 & $8.536,8$ & 285,1 & 189,4 & 180,3 & 245,2 & 220,7 \\
\hline & 10 & $8.453,5$ & 322,0 & 246,5 & 197,5 & 294,7 & 268,2 \\
\hline
\end{tabular}

Quadro 5. Teores médios de K trocável e não-trocável após o cultivo do feijoeiro, quantidades de potássio li beradas e absorvidas durante o experimento, considerando a saturação por $K$

\begin{tabular}{|c|c|c|c|c|c|c|c|}
\hline Solo & Saturação de K & Final & Liberado & Final & Liberado & K total liberado & K absorvido \\
\hline & $\%$ & $\mathrm{mg} \mathrm{dm}^{-3}$ & mg vaso-1 & $\mathrm{mg} \mathrm{dm}^{-3}$ & 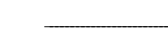 & mg vaso $^{-1}$ & \\
\hline OY & $\begin{array}{c}\text { Natural } \\
4 \\
6 \\
8 \\
10\end{array}$ & $\begin{array}{c}24,6(77)^{(1)} \\
38,3(72) \\
48,1(76) \\
71,2(71) \\
104,2(66)\end{array}$ & $\begin{array}{l}251,0 \\
301,6 \\
450,9 \\
539,2 \\
602,6\end{array}$ & $\begin{array}{l}63,0(34)^{(1)} \\
63,1(36) \\
71,2(46) \\
62,4(62) \\
67,4(69)\end{array}$ & $\begin{array}{r}98,6 \\
106,6 \\
179,0 \\
303,1 \\
443,5\end{array}$ & $\begin{array}{r}349,6 \\
408,2 \\
624,9 \\
842,3 \\
1046,1\end{array}$ & $\begin{array}{l}428,4(122)^{(2)} \\
471,4(115) \\
656,9(104) \\
790,4(94) \\
869,4(83)\end{array}$ \\
\hline GM & $\begin{array}{c}\text { Natural } \\
4 \\
6 \\
8 \\
10\end{array}$ & $\begin{array}{r}36,2(69) \\
42,1(77) \\
76,2(72) \\
89,5(76) \\
134,2(72)\end{array}$ & $\begin{array}{r}238,8 \\
414,7 \\
583,1 \\
840,5 \\
1017,4\end{array}$ & $\begin{array}{r}16,0(76) \\
22,2(77) \\
40,0(76) \\
48,9(78) \\
110,9(55)\end{array}$ & $\begin{array}{l}147,9 \\
218,4 \\
377,0 \\
516,6 \\
405,1\end{array}$ & $\begin{array}{r}386,7 \\
633,1 \\
960,1 \\
1357,1 \\
1422,5\end{array}$ & $\begin{array}{c}357,8(92) \\
716,9(113) \\
1033,9(107) \\
1293,5(95) \\
1402,9(98)\end{array}$ \\
\hline $\mathrm{GX}$ & $\begin{array}{c}\text { Natural } \\
4 \\
6 \\
8 \\
10\end{array}$ & $\begin{array}{l}11,4(53) \\
14,8(69) \\
23,2(67) \\
35,6(60) \\
46,4(63)\end{array}$ & $\begin{array}{r}38,3 \\
100,2 \\
142,5 \\
159,5 \\
235,3\end{array}$ & $\begin{array}{l}16,9(28) \\
20,0(49) \\
18,0(65) \\
11,1(81) \\
23,5(70)\end{array}$ & $\begin{array}{r}20,3 \\
59,0 \\
100,0 \\
140,1 \\
167,5\end{array}$ & $\begin{array}{r}55,2 \\
159,2 \\
242,5 \\
299,6 \\
402,8\end{array}$ & $\begin{array}{l}152,8(277) \\
260,7(163) \\
304,0(125) \\
437,6(146) \\
536,1(133)\end{array}$ \\
\hline$R U$ & $\begin{array}{c}\text { Natural } \\
4 \\
6 \\
8 \\
10\end{array}$ & $\begin{array}{l}20,8(68) \\
32,4(66) \\
45,6(68) \\
53,0(72) \\
62,8(75)\end{array}$ & $\begin{array}{l}131,9 \\
191,5 \\
296,0 \\
409,1 \\
550,9\end{array}$ & $\begin{array}{l}176,1(26) \\
175,4(38) \\
177,0(36) \\
188,2(34) \\
204,9(36)\end{array}$ & $\begin{array}{l}184,7 \\
318,1 \\
292,2 \\
290,5 \\
351,3\end{array}$ & $\begin{array}{l}316,6 \\
509,6 \\
588,2 \\
699,6 \\
902,2\end{array}$ & $\begin{array}{l}241,2(76) \\
377,3(74) \\
531,3(90) \\
751,2(107) \\
829,0(92)\end{array}$ \\
\hline
\end{tabular}

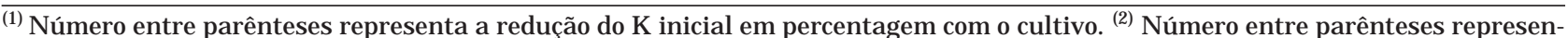
ta a percentagem do K absorvido em relação ao K liberado total. 
também se correlacionou positivamente com a quantidade de K não-trocável liberada (Quadro 6).

Quanto à produção do feijoeiro, observou-se grande variação dos valores estimados para a saturação por K, entre os solos (Quadro 7). Para $90 \%$ da produção máxima, a menor saturação por K observada para osolo GM, de 4,1 \%, foi justificada pelos maiores incrementos na produção com as primeiras doses de $\mathrm{K}$ (saturações), o que resultou num ajuste do modelo raiz quadrática aos dados. Da mesma maneira, justificou-se o alto val or para a saturação deK no sol o GX, de 8,7 \%, em decorrência do ajuste linear dos dados (Quadro 7).

Para a obtenção de 90 \% da produção máxima, os teores de $\mathrm{K}$ disponível, tanto pelos extratores Mehlich-1 quanto pela Resina, não apresentaram grande variação entre os solos (Quadro 8). No entanto, para esse mesmo nível de produção, as saturações por K da CTC potencial variaram proporcionalmente, mais de 4,1 a 8,7 \% (Quadro 7), bem como as doses de K aplicadas para atingir essas saturações, 83 a $155 \mathrm{mg} \mathrm{dm}^{-3}$ (Quadro 9). Assim, a recomendação de adubação potássica, objetivando atingir determinada faixa de saturação da CTC pelo nutriente, não pode ser generalizada. Neste caso, seria necessário estabelecer classes de valores de CTC, com recomendação específica de adubação potássica para cada classe, visando atingir os val ores ideais de saturação por K para determinada cultura.

As plantas defeijoei ro apresentaram maior nível crítico de K, quando cultivadas no solo GM (Quadro 10), que dispõe do maior poder tampão desseelemento (Quadro 3). Para oP, segundo Muniz et al. (1985), a facilidade de absorção do nutriente da solução que está em equilíbrio com a forma lábil, pelas plantas, é negativamente relacionada com o poder tampão deste el emento no solo. Desse modo, em sol os com maior poder tampão, há uma menor absorção de $\mathrm{P}$ pelas plantas. Do mesmo modo que, segundo Holford \& Mattingly (1976), em solos com o mesmo fator intensidade, as plantas absorvem $P$ em quantidade diretamente proporcional ao fator capacidade de $\mathrm{P}$ do solo, em virtude do maior poder de reposição do $P$ à solução, em conseqüência da absorção pelas raízes. Então, deforma semelhante, o maior nível crítico de $\mathrm{K}$ observado nas plantas cultivadas no solo GM pode ser explicado pel o fato de o sol o necessitar de maior quantidade de K para saturar o complexo detroca, daí a maior capaci dade de repor o K na solução quando este é absorvido. Assim sendo, as plantas cultivadas no GM em relação às cultivadas nos demais sol os tinham, durantetodo o período de cultivo, maior disponibilidade de $\mathrm{K}$, o que justifica o maior nível crítico.

Quadro 6. Coeficientes de correlação entre potássio trocável (Kt) inicial, final e liberado; potássio nãotrocável (Knt) inicial, final e liberado; potássio absorvido(K abs) e matéria seca de grãos (MSGR)

\begin{tabular}{|c|c|c|c|c|c|c|c|c|}
\hline & Kt inicial & Kt final & Kt liberado & Knt inicial & Knt final & Knt liberado & K Abs & MSGR \\
\hline Kt inicial & - & $0,97 * *$ & $0,99 * *$ & $0,52 * *$ & $0,16 \mathrm{~ns}$ & $0,85^{* *}$ & $0,87 * *$ & $0,79 * *$ \\
\hline Kt final & - & - & $0,95^{* *}$ & $0,50 * *$ & $0,14 \mathrm{~ns}$ & $0,84 * *$ & $0,87 * *$ & $0,72 * *$ \\
\hline Kt liberado & - & - & - & $0,51^{* *}$ & $0,16 n s$ & $0,84 * *$ & $0,86 * *$ & $0,81 * *$ \\
\hline Knt inicial & - & - & - & - & $0,90 * *$ & $0,76 * *$ & $0,52 * *$ & $0,28^{\text {ns }}$ \\
\hline Knt final & - & - & - & - & - & $0,40 *$ & 0,21 ns & $-0,04^{n s}$ \\
\hline Knt liberado & - & - & - & - & - & - & $0,78 * *$ & $0,64 * *$ \\
\hline $\mathrm{K}$ abs. & - & - & - & - & - & - & - & $0,80 * *$ \\
\hline
\end{tabular}

**, * e ns significativo a 1, 5 \% e não significativo, respectivamente.

Quadro 7. Equações de regressão ajustadas para a matéria seca de grãos (y em $\left.\mathrm{g} \mathrm{vaso}^{-1}\right)$, considerando a saturação por potássio ( $x$ em \%) e estimativas de $90 \%$ da matéria seca e as saturações por potássio estimadas para promover essas produções

\begin{tabular}{|c|c|c|c|c|c|c|c|}
\hline \multirow{2}{*}{ Solo } & \multirow{2}{*}{\multicolumn{2}{|c|}{ E quação }} & \multirow{2}{*}{$\mathbf{R}^{2}$} & \multicolumn{2}{|c|}{ MSGR (g vaso-1) } & \multicolumn{2}{|c|}{ Saturação por K (\%) } \\
\hline & & & & $90 \%$ & Máxima & $90 \%$ & Máxima \\
\hline OY & $\hat{\mathrm{y}}$ & $=-7,4913+10,7661 * * x-0,7662 * * x^{2}$ & 0,94 & 27,3 & 30,3 & $5,0(71)^{(1)}$ & 7,0 \\
\hline GM & $\hat{\mathrm{y}}$ & $=-31,9698+47,5491 * * x^{0,5}-8,9645^{* *} x$ & 0,90 & 28,0 & 31,1 & $4,1(59)$ & 7,0 \\
\hline$G X$ & $\hat{y}$ & $=5,5715+1,3344 * * x$ & 0,91 & 15,9 & 17,7 & $8,7(87)$ & 10,0 \\
\hline RU & $\hat{y}$ & $=1,0485+4,9431 * * x-0,3161 * * x^{2}$ & 0,96 & 18,4 & 20,4 & $5,3(68)$ & 7,8 \\
\hline
\end{tabular}

(1) Percentagem em relação à saturação máxima, necessária para atingir 90 \% da máxima produção. 
Quadro 8. Equações de regressão ajustadas para o K disponível pelos extratores Mehlich-1 e Resina (y em $\mathrm{mg} \mathrm{dm}^{-3}$ ) de acordo com a saturação por $\mathrm{K}$ da CTC potencial (x em \%)

\begin{tabular}{|c|c|c|c|c|c|}
\hline \multirow{2}{*}{ Solo } & \multirow{2}{*}{\multicolumn{2}{|c|}{ E quação }} & \multirow{2}{*}{$\mathrm{R}^{2}$} & \multicolumn{2}{|c|}{ Teor de $\mathrm{K}\left(\mathrm{mg} \mathrm{dm}^{-3}\right)$} \\
\hline & & & & $90 \%$ & Máxima \\
\hline \multicolumn{6}{|c|}{ Mehlich-1 } \\
\hline OY & $\hat{y}$ & $=37,5525+30,0795^{* *} x$ & 0,99 & 188 & 248 \\
\hline GM & $\hat{y}$ & $=9,4681+39,4170 * * x$ & 0,99 & 171 & 285 \\
\hline$G X$ & $\hat{y}$ & $=0,6319+21,7893 * * x$ & 0,99 & 190 & 218 \\
\hline $\mathrm{RU}$ & $\hat{\mathrm{y}}$ & $=28,5234+26,9206 * * x$ & 0,99 & 171 & 238 \\
\hline \multicolumn{6}{|c|}{ Resina } \\
\hline OY & $\hat{\mathrm{y}}$ & $=-0,8549+37,3410 * * x$ & 0,99 & 186 & 260 \\
\hline GM & $\hat{y}$ & $=-9,0236+44,5790 * * x$ & 0,99 & 173 & 303 \\
\hline $\mathrm{GX}$ & $\hat{\mathrm{y}}$ & $=0,9129+19,4469 * * x$ & 0,99 & 170 & 195 \\
\hline $\mathrm{RU}$ & $\hat{\mathrm{y}}$ & $=13,3904+25,8980 * * x$ & 0,99 & 150 & 215 \\
\hline
\end{tabular}

** Significativo a $1 \%$.

Quadro 9. Equações de regressão ajustadas para o K disponível pelos extratores Mehlich-1 e Resina (y em $\mathrm{mg} \mathrm{dm}^{-3}$ ) de acordo com as quantidades de $\mathrm{K}$ aplicadas aos solos ( $\mathrm{em} \mathrm{mg} \mathrm{dm}^{-3}$ )

\begin{tabular}{|c|c|c|c|c|c|}
\hline \multirow{2}{*}{ Solo } & \multirow{2}{*}{\multicolumn{2}{|c|}{ E quação }} & \multirow{2}{*}{$\mathrm{R}^{2}$} & \multicolumn{2}{|c|}{ Dose de $\mathrm{K}\left(\mathrm{mg} \mathrm{dm}^{-3}\right)$} \\
\hline & & & & $90 \%$ & Máxima \\
\hline \multicolumn{6}{|c|}{ Mehlich-1 } \\
\hline OY & $\hat{y}$ & $=137,3640+0,7173^{* *} x$ & 0,99 & 70 & 154 \\
\hline GM & $\hat{y}$ & $=111,4450+0,7199 * * x$ & 0,99 & 83 & 241 \\
\hline GX & $\hat{\mathrm{y}}$ & $=43,6961+0,9454 * * x$ & 0,99 & 155 & 184 \\
\hline $\mathrm{RU}$ & $\hat{\mathrm{y}}$ & $=91,7121+0,7760 * * x$ & 0,99 & 102 & 180 \\
\hline \multicolumn{6}{|c|}{ Resina } \\
\hline OY & $\hat{\mathrm{y}}$ & $=123,0490+0,8991 * * x$ & 0,99 & 70 & 152 \\
\hline GM & $\hat{\mathrm{y}}$ & $=106,3070+0,8142 * * x$ & 0,99 & 82 & 241 \\
\hline GX & $\hat{\mathrm{y}}$ & $=39,3247+0,8440 * * x$ & 0,99 & 155 & 184 \\
\hline RU & $\hat{\mathrm{y}}$ & $=74,2206+0,7462 * * x$ & 0,99 & 99 & 189 \\
\hline
\end{tabular}

** Significativo a $1 \%$.

Quadro 10. Equações de regressão ajustadas para os teores foliares de $K\left(\mathrm{y} \mathrm{em} \mathrm{g} \mathrm{kg}^{-1}\right)$ na época do florescimento, como variável dependente das saturações por $K$ estabelecidas nos tratamentos (x em \%) e níveis críticos inferiores (para 90 \% da MSGR máxima)

\begin{tabular}{|c|c|c|c|c|}
\hline Solo & & E quação & $\mathbf{R}^{2}$ & $\begin{array}{c}\text { Nível crítico } \\
\text { inferior }\left(\text { g kg-1) }^{-1}\right)\end{array}$ \\
\hline OY & $\hat{y}$ & $=11,9835+1,1856 * * x$ & 0,87 & 17,9 \\
\hline GM & $\hat{\mathrm{y}}$ & $=14,0477+4,0131 * * x$ & 0,91 & 30,5 \\
\hline $\mathrm{GX}$ & $\hat{y}$ & $=10,5191+0,7203^{* *} \mathrm{x}$ & 0,81 & 16,8 \\
\hline $\mathrm{RU}$ & $\hat{y}$ & $=2,7478+2,2385^{* *} x$ & 0,96 & 14,6 \\
\hline
\end{tabular}

** Significativo a $1 \%$.

\section{CONCLUSÕES}

1. O Organossolo Mésico, Gleissolo Melânico, Gleissol o Háplico e N eossol o Flúvico diferiram entre si quanto às diferentes formas de $\mathrm{K}$ avaliadas. $\mathrm{O}$ Organossol o Mésicoe Neossol oFlúvico apresentaram maiores teores deK nas formas: total enão-trocável.

2. A forma deK trocável foi a que mais contribuiu para a absorção desse el emento pel o feijoeiro.

3. A produção do feijoeiro foi significativamente aumentada com a aplicação de K, apesar dos teores médios e altos de $\mathrm{K}$ disponível nos solos.

4. As saturações críticas por $K$ da capacidade de troca de cátions potencial correspondentes a $90 \%$ 
da produção produção máxima foram de 5,0, 4,1, 8,7 e 5,3 \%, para os sol os Organossolo Mésico, Gleissolo Melânico, Gleissolo Háplico e Neossolo Flúvico, respectivamente.

5. Os níveis críticos para a concentração de K nos tecidos foliares do feijoeiro na época do flor escimento foram de 17,9, 30,5, 16,8 e 14,6 $\mathrm{mg} \mathrm{dm}^{-3}$, para Organossolo Mésico, Gleissolo Melânico, Gleissolo Háplico e Neossolo Flúvico, respectivamente.

\section{LITE RATURA CITADA}

BECKETT, P.H.T. Studies on soil potassium. II. The "imediated" Q/I relations of labile potassium in the soil. J. Soil Sci., 15:9-23, 1964.

BECKETT, P.H.T. \& NAFADY, M.H.M. Studies on soil potassium. VI. The effect of $\mathrm{K}$-fixation and release on the form of the $\mathrm{K}:(\mathrm{Ca}+\mathrm{Mg})$ exchange isotherm. J . Soil Sci., 18:244-262, 1967.

CHAVES, L.H.G. \& KINJ O, T. Relação quantidade/intensidade de potássio em solos do trópico semi-árido brasileiro. R. Bras. Ci. Solo, 11:257-261, 1987.

COMISSÃO DE FERTILIDADE DO SOLO DO ESTADO DE MINAS GERAIS - CFSEMG. Recomendações para o uso de corretivos e fertilizantes em Minas Gerais. Viçosa, 1999. 359p.

EMPRESA BRASILEIRA DE PESQUISA AGROPECUÁRIA EMBRAPA. Centro Nacional de Pesquisa de Solos. Manual de métodos e análises de solos, 2.ed. Rio de J aneiro, 1997. 212p. (Documentos, 1)

GIANELO, C. \& MIELNICZUK, J. Características químicas e físicas do sol o que afetam a absorção de potássio por plantas de milho. R. Bras. Ci. Solo, 5:109-114, 1981.

GOEDERT, W.J .; SYER, J.K. \& COREY, R.B. Relações quantidade - intensidade de potássio em solo do Rio Grande do Sul. Pesq. Agropec. Bras., 10:31-35, 1975.

HOLFORD, I.C.P. \& MATTINGLY, G.E.G. Phosphate adsorption and plant availability of phosphorus. Plant Soil, 44:377-389, 1976.

KNUDSEN, D.; PETERSON, G.A. \& PRATT, P.F. Lithium, sodium and potassium. In: PAGE, A.L., ed. Methods of soil analisys; chemical and microbiological properties. Madison, American Society of Agronomy/Soil Science Society of America, 1982. p.225-246.
MALAVOLTA, E.; VITTI, G.C. \& OLIVEIRA, S.A. Avaliação do estado nutricional das plantas: princípios e aplicações. Piracicaba, Associação Brasileira para Pesquisa da Potassa e do F osfato, 1997. 319p.

MIELNICZUK, J. O potássio no solo. Piracicaba, Instituto de Potassa e do Fosfato, 1978. 80p. (Boletim Técnico, 2)

MIELNICZUK, J . Avaliação da resposta das culturas ao potássio em ensaios de longa duração, experiências brasileiras. In: YAMADA, T.; MUZZILLI, O. \& USHERWOOD, N.R., eds. Potássio na agricultura brasileira. Piracicaba, Instituto da Potassa eF osfato. Instituto I nternacional da Potassa, 1982. p.289-303.

MIELNICZUK, J . \& SELBACH,P.A. Capacidade de suprimento de potássio de seis solos do Rio Grande do Sul. R. Bra. Ci. Solo, 2:115-120, 1978.

MUNIZ, A.S.; NOVAIS, R.F.; BARROS, N.F. \& NEVES, J.C.L. Nível crítico de fósforo na parteaérea da soja como variável do fator capacidade de fósforo do solo. R. Bras. Ci. Solo, 9:237-243, 1985

NACHTIGALL, G.R. \& VAHL, L.C. Formas de potássio em solos da região sul do Estado do Rio Grande do Sul. R. Bras. Ci. Solo, 13:7-12, 1989.

PREZOTTI, L.C. \& DEFELIPO, B.V. Formas de potássio em sol os do Estado de Minas Gerais. R. Bras. Ci. Solo, 11:109114, 1987.

RAIJ , B.van. Avaliação da fertilidade do solo. Piracicaba, Instituto da Potassa e F osfato - Instituto I nternacional da Potassa, 1981. 142p.

RAIJ , B. van; QUAGGIO, J .A.; CANTARELLA, H.; FERREIRA, M.E.; LOPES, A.S. \& BATAGLIA, O.C. Análise química do solo para fins defertilidade. Campinas, Fundação Cargil, 1987. 170p.

REITEMEIER, R.F. The chemistry of soil potassium. Adv. Agron., 3:133-164, 1951.

RICH, C.I. Mineralogy of soil potassium. In: KILMER, V.J .; YOUNTS, S.E. \& BRADY, N.C., eds. The role of potassium in agriculture. Madison, Soil Science Society of American, 1968. p.79-108.

SILVA, I.R.; FURTINI NETO, A.E.; FERNANDES, L.A.; CURI, N. \& VALE, F.R. Formas, relação quantidade/ intensidade e biodisponibilidade de potássio em diferentes Latossolos. Pesq. Agropec. Bras., 35:2065-2073, 2000.

SPARKS, D.L. Chemistry of soil potassium in Atlantic Coastal Plain soils: a review. Comm. Soil Sci. Plant Anal., 11:435449, 1980.

TISDALE, S.L. \& NELSON, W.L. Soil fertility and fertilizers. 5.ed. New York, Macmillan, 1993. 634p. 